\title{
EBSD study of advanced Ti-5.7Al-3.8Mo-1.2Zr-1.3Sn alloy subjected to equal-channel angular pressing
}

\author{
G.S. Dyakonov ${ }^{a, *}$, T.V. Yakovleva ${ }^{a}$, A.G. Stotskiy ${ }^{a}$, S. Mironov ${ }^{b}$ \\ a Institute of Physics of Advanced Materials, Ufa State Aviation Technical University, 12 K. Marx str., Ufa 450008, Russia \\ ${ }^{\mathrm{b}}$ Laboratory of Mechanical Properties of Nanoscale Materials and Superalloys, Belgorod National Research University, 85 Pobeda str., Belgorod 308015, Russia
}

\section{A R T I C L E I N F O}

\section{Article history:}

Received 28 February 2021

Received in revised form 3 April 2021

Accepted 2 May 2021

Available online 5 May 2021

\section{Keywords:}

Titanium alloys

Equal-channel angular pressing (ECAP)

Microstructure

Texture

Electron backscatter diffraction (EBSD)

\begin{abstract}
A B S T R A C T
In this work, electron backscatter diffraction (EBSD) was applied for microstructural characterization of the advanced Ti-5.7Al-3.8Mo-1.2Zr-1.3Sn alloy subjected to equalchannel angular pressing (ECAP). It was found that the spheroidization process induced by ECAP was governed by the conventional boundarysplitting mechanism. The nearly-Burgers orientation relationship between the spheroidized a- and bphases was revealed. This intriguing result can be attributed to the very high cooperation in slip activity between the phases.
\end{abstract}

(c) 2021 Elsevier B.V. All rights reserved.

\section{Introduction}

Ti-5.7Al-3.8Mo-1.2Zr-1.3Sn alloy is an advanced material, which has been developed recently as a substitute for Ti-6Al-4V in high-temperature applications [1]. Due to the relatively high fraction of $\beta$-phase and precipitation of intermetallic dispersoids, this alloy demonstrates an excellent thermal stability at the temperatures up to $\sim 450-500{ }^{\circ} \mathrm{C}$. Therefore, the application of this material instead of Ti-6Al-4V will enhance performance of the engine compressor [2]. For industrial application of this material, however, a comprehensive examination of its mechanical properties and microstructural behavior are necessary. At the present time, however, the studies in this area are limited to a few examples [3-6].

In our previous study [3], equal-channel angular pressing (ECAP) has been applied for the first time for grain refinement of the new alloy. This approach was found to be very effective for production of the ultrafine-grained structure with the mean grain size of $\sim 0.5 \mu \mathrm{m}$. Attempting to provide deeper insight into the microstructural behavior of the new alloy during ECAP, electron backscatter diffraction (EBSD) was applied in the present work.

\footnotetext{
* Corresponding author.

E-mail address: dgr84@mail.ru (G.S. Dyakonov).
}

\section{Material and methods}

The initial material was provided by VSMPO-AVISMA Corporation (Russia) and supplied as a hot extruded bar. The $\beta$ transus temperature of this alloy was measured to be $980^{\circ} \mathrm{C}$. Prior to ECAP, the received alloy underwent a two-stage heat-treatment, which involved a quenching in water from $940{ }^{\circ} \mathrm{C}$, and subsequent annealing at $700{ }^{\circ} \mathrm{C}$ for $1 \mathrm{~h}$ followed by air cooling. From the heat-treated material, the billets of $20 \mathrm{~mm}$ in diameter and $150 \mathrm{~mm}$ in length were machined, and subjected to 4 ECAP passes using $B_{C}$ route. The details of the ECAP procedure have been described in the previous work [3]. Briefly, ECAP was conducted at a speed of $4 \mathrm{~mm} / \mathrm{s}$ using a die with a $120^{\circ}$ round channel. The total accumulated true strain was evaluated to be 2.8 . To provide high-temperature deformation conditions, the working billets were held at $750{ }^{\circ} \mathrm{C}$ for 30 min before each pass; moreover, the die was heated to $550{ }^{\circ} \mathrm{C}$ in order to minimize material cooling during ECAP. Following the final ECAP pass, the processed material was quenched in water.

Microstructural observations were performed primarily by EBSD but also involved both scanning (SEM) and transmission electron microscopy (TEM). The details of SEM and TEM experiments have been described elsewhere [3]. EBSD specimens were prepared using conventional metallographic techniques followed by 24-h vibratory polishing with colloidal silica suspension. EBSD was conducted with a Hitachi S-4300SE field emission gun scanning electron microscope operated at $25 \mathrm{kV}$ and equipped with a TSL 

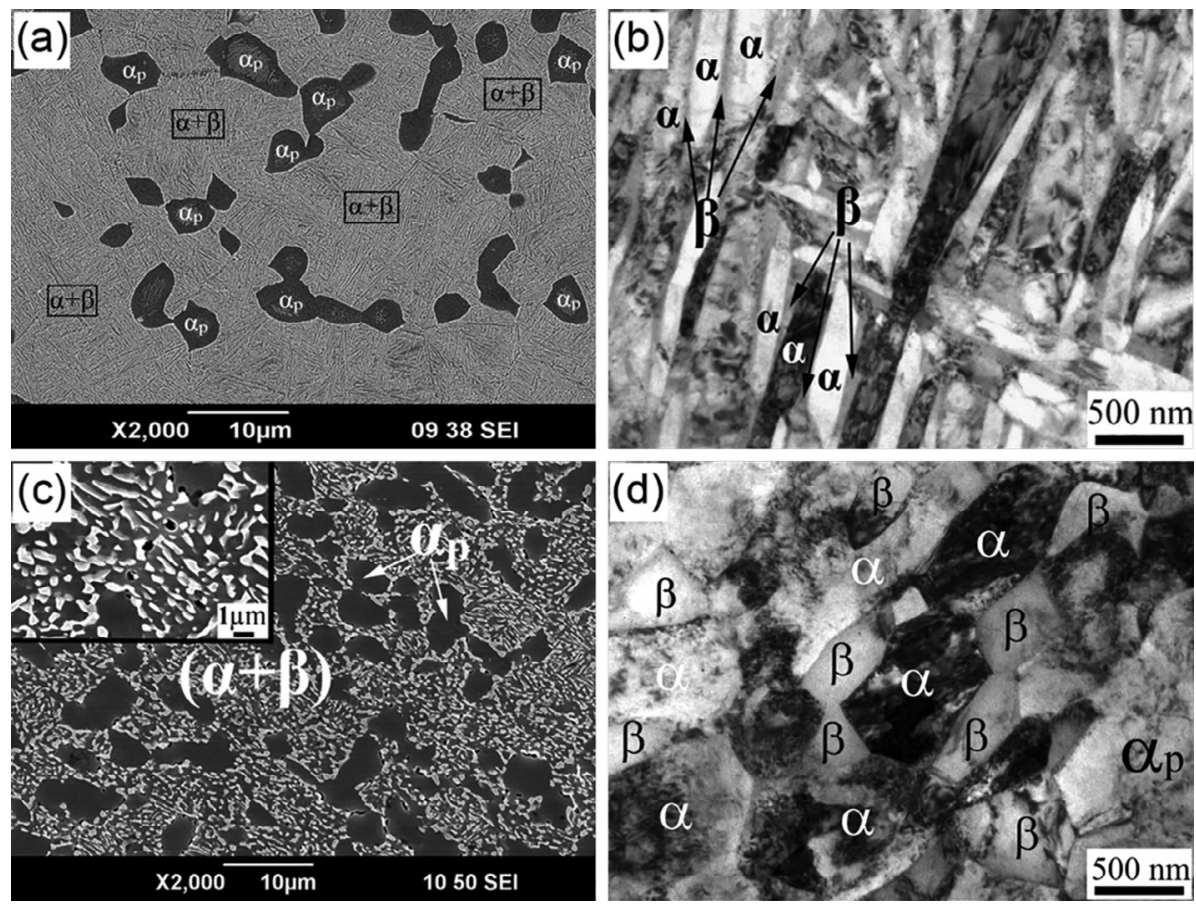

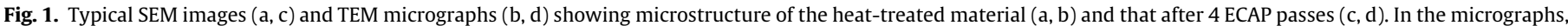

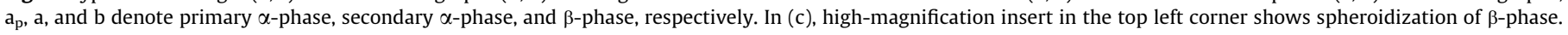

$\mathrm{OIM}^{\mathrm{TM}}$ EBSD system. The scan step size was $0.5 \mu \mathrm{m}$. To improve reliability of EBSD data, the grains comprising 3 or fewer pixels were automatically removed from EBSD using the standard grain-dilation option. A $15^{\circ}$ tolerance was applied to differentiate low-angle boundaries (LABs) from high-angle boundaries (HABs).

\section{Results and discussion}

\subsection{Microstructure morphology and grain size}

The initial microstructure is shown in Fig. 1a and b. The applied heat treatment resulted in the formation of a duplex microstructure dominated by relatively fine transformed $\beta$-grains with a comparatively small fraction ( $20 \%)$ of primary $\alpha$-phase (Fig. 1a). As expected, the transformed $\beta$-microstructure was comprised of relatively fine $(\approx 0.12 \mu \mathrm{m}$ in width) $\alpha$ - and $\beta$-lamellae (Fig. 1b).

ECAP led to the essential fragmentation of a lamellar microstructure (Fig. 1c and d). Moreover, the fragmented $\alpha$ - and $\beta$-lamellae took a nearly-equiaxed shape and grew up to $\sim 0.5 \mu \mathrm{m}$ (Fig. 1d), which points to an activation of the spheroidization process. Remarkably, both $\alpha$ - and $\beta$-phases had a relatively high dislocation density (Fig. 1d). At the same time, the primary $\alpha$-phase underwent no substantial changes (Fig. 1c).

EBSD phase map taken from the ECAP-processed material is shown in Fig. 2a. The grain size distribution derived from the EBSD data is shown in Fig. 2b. The specific character of the grain-size distribution in the $\alpha$-phase which contained a sharp peak near $6 \mu \mathrm{m}$ and a broad maximum centered near $3 \mu \mathrm{m}^{1}$ indicated a formation of the bimodal grain structure. Remarkably, the fine $\alpha$-grains were preferentially concentrated near $\beta$-particles (Fig. 2a). Hence, it can be inferred that a grain refinement in the $\alpha$-phase took place mainly within the lamellar microstructure. On the other hand, the primary

\footnotetext{
1 The apparent discrepancy in grain size measurements between EBSD and TEM was associated with the formation of the developed LAB structure within both phases, as shown in Section 3.2. Specifically, the structural elements revealed by TEM in Fig. 1d likely represented subgrains.
}

$\alpha$-phase appeared to be relatively stable against the strain. The relatively high grain-refinement rate revealed in the lamellar microstructure is thought to be related with the influence of the $\beta$ phase. To provide strain compatibility of the adjoining $\alpha$ and $\beta$ phases, a high density of the geometrically-necessary dislocations was presumably generated which enhanced the microstructure transformation process.

\subsection{Misorientation distribution}

In order to get an additional insight into microstructural processes, misorientation distributions were examined. To investigate this matter more thoroughly, distributions of each microstructural component were measured, including both fine-grained and coarse-grained $\alpha$-phase, and $\beta$-phase, as shown in Fig. $2 c-e$, respectively.

A relatively large proportion of LABs fraction in fine-grained $\alpha$ and $\beta$-phases (Fig. 2c and e) agrees well with rather high dislocation density revealed in these microstructural components by TEM (Fig. 1d). Eventually, these observations imply that a spheroidization process mainly resulted from the formation of deformation-induced boundaries, i.e. it was predominantly governed by a conventional boundary-splitting mechanism [7]. The details of this process are not clear, however, and require further study.

At the same time, an extremely high fraction of LABs in a coarse-grained $\alpha$-phase (Fig. $2 \mathrm{~d}$ ) indicated that this microstructural component experienced a substantial plastic strain. The presence of transient misorientations in the spectrum (i.e., $10-20^{\circ}$ ) evidenced the LAB-to-HAB transformation to occur. This virtually implied the activation of the continuous recrystallization mechanism. However, the intensity of this process in the primary $\alpha$ was surprisingly low. Hence, it can be suggested that the material flow during ECAP was preferentially concentrated in the transformed fine-grained material whereas the coarse-grained primary $\alpha$ experienced only an accommodation strain. 

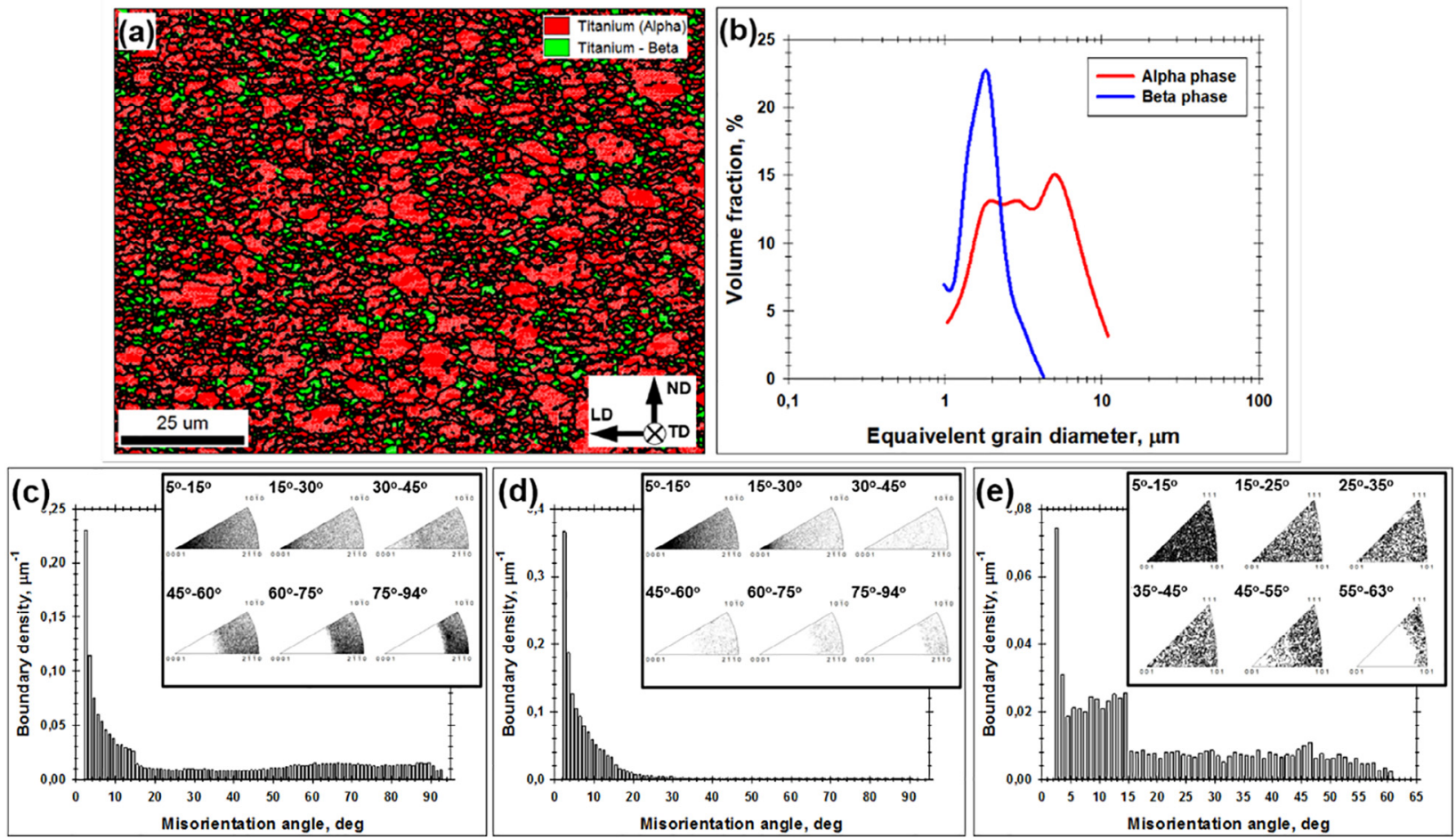

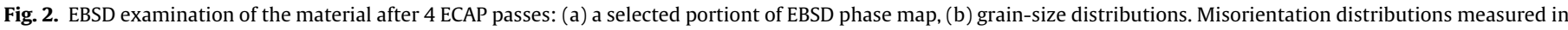

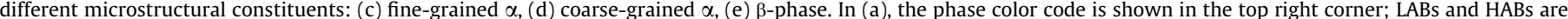

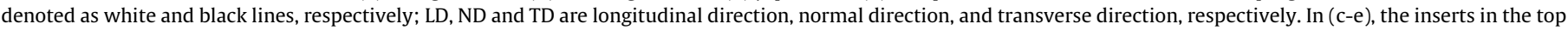
right corners show misorientation-axis distributions.

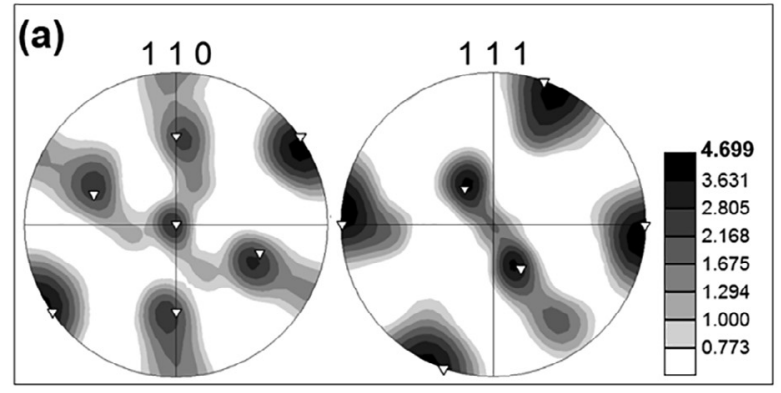

Texture Name: Harmonic: $L=16, H W=5.0$ Calculation Method: Harmonic Series Expansion

Series Rank (l): 16

Gaussian Smoothing: $5.0^{\circ}$

Sample Symmetry. Triclinic

$O P_{1}\{\overline{1} 100\}<\overline{2} 110>$

---- P-fiber $\{h k i\}<11 \overline{2} 0>$

$\diamond B$-fiber $\{0001\}<$ uvtw>

« C-fiber

$\nabla D_{2}\{11 \overline{2}\}<111>$
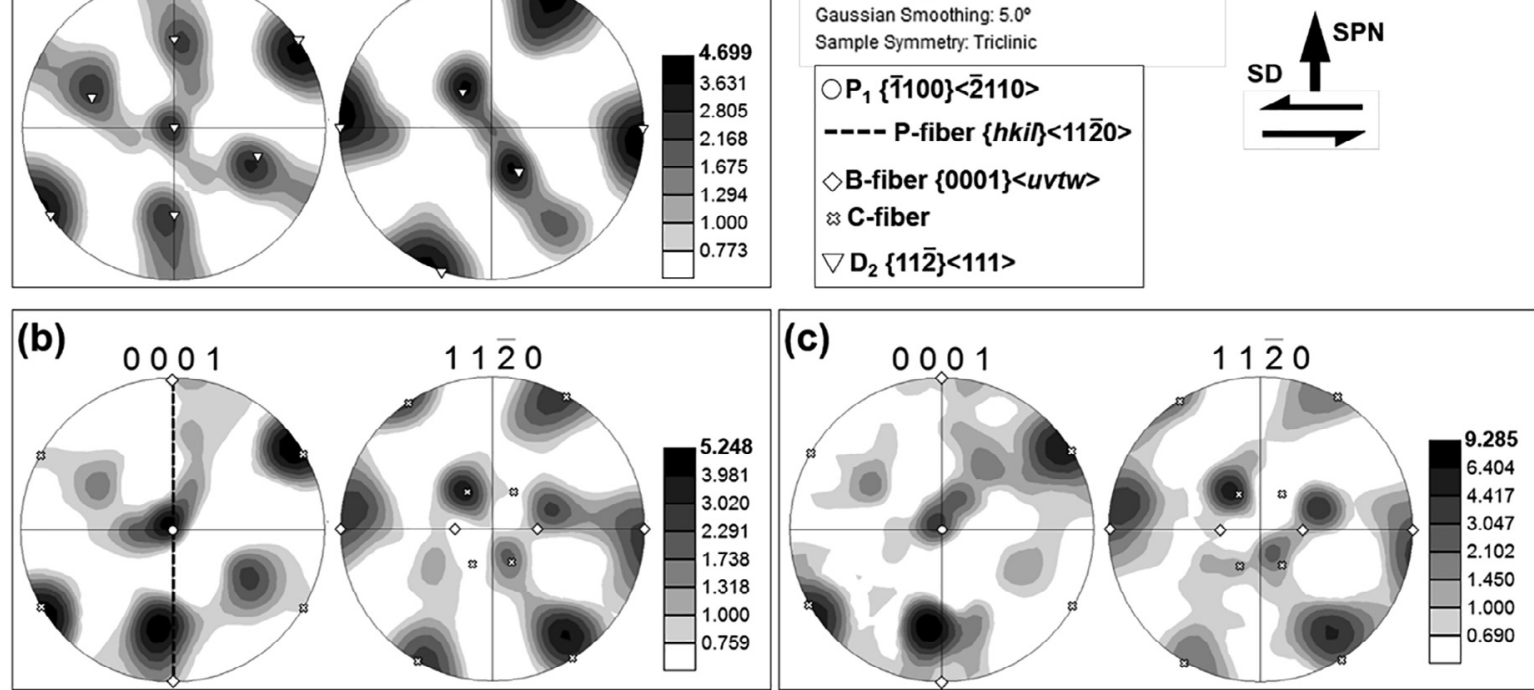

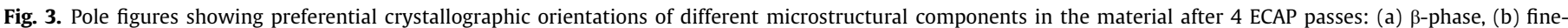
grained $\alpha$, and (c) coarse-grained $\alpha$. SD and SPN denote shear direction and shear plane normal, respectively.

\subsection{Texture}

To examine slip activity during ECAP, orientation data were derived from the EBSD map, arranged as pole figures and summarized in Fig. 3. It is important to emphasize, that the measured pole figures were appropriately rotated in order to align their reference frame with the presumed geometry of simple shear during ECAP, as discussed by Li [8]. For simplicity, some ideal simple-shear orientations were superimposed on the pole figures.

One of the most striking observations was a close similarity between $\beta$-phase and fine-grained $\alpha$-phase texture patterns (compare Fig. 3a and b). This result can be explained by the "global" 
Burgers orientation relationship between the phases, viz. $\{110\}_{\beta} / /\{0001\}_{\alpha}$ and $<111>_{\beta} / /<11 \overline{2} 0>_{\alpha}$.

Considering the relatively low deformation temperature $\left(\leq 750{ }^{\circ} \mathrm{C}\right)$, no essential $\beta \rightarrow \alpha$ phase transformation was expected to occur during material cooling, and therefore the observed effect cannot be attributed to a transformation texture. If so, this phenomenon appears to imply a very close cooperation in slip activity within $\alpha$-and $\beta$-phases during ECAP.

In $\beta$-phase, the texture was dominated by the $D_{2}\{11 \overline{2}\}<111>$ simple shear orientation (Fig. 3a), which presumably implied the prevalence of $\{112\}$ slip during ECAP. The fine-grained $\alpha$-phase was characterized by the relatively complex texture which included $\quad P_{1}\{\overline{1} 100\}<\overline{2} 110>\quad$ component, $P$-fiber $\{h k i l\}<11 \overline{2} 0\rangle, B$-fiber $\{0001\}<u v t w>$, and C-fiber (Fig. 3b). According to $\mathrm{Li}$ [8], the formation of this texture is attributable to the combined effect of the prism-, basal-, and pyramidal $\langle c+a\rangle$ slip modes. It is particularly remarkable, that nearly the same texture was also found in a coarse-grained $\alpha$-phase (compare Fig. 3c and b). This slip activity is thought to be associated with relatively high deformation temperature.

\section{Conclusions}

It was suggested, that the spheroidization process is governed by the conventional boundary-splitting mechanism. On the other hand, the evolution of a primary $\alpha$-phase was found to be driven by a continuous recrystallization.

Surprisingly, a nearly-Burgers orientation relationship between the spheroidized $\alpha$-and $\beta$-phases was found. This unusual result was attributed to the very close cooperation in slip activity within the $\alpha$-and $\beta$-phases.

Texture analysis showed the prevalence of $\{112\}$ slip in the $\beta$ phase. In the $\alpha$-phase, material flow resulted from the combined effect of the prism-, basal- and pyramidal $\langle c+a\rangle$ slip modes.

Despite the present study having provided a useful insight into microstructural evolution of the new alloy during ECAP, the key mechanisms of this process are still unclear and warrants further study.

\section{CRediT authorship contribution statement}

G.S. Dyakonov: Conceptualization, Data curation, Investigation. T.V. Yakovleva: Visualization, Investigation. A.G. Stotsky: Formal analysis, Resources, Investigation. S. Mironov: Methodology, Validation, Investigation, Writing - original draft.

\section{Declaration of Competing Interest}

The authors declare that they have no known competing financial interests or personal relationships that could have appeared to influence the work reported in this paper.

\section{Acknowledgment}

The reported study was funded by RFBR, project number No. 20-38-70105.

\section{References}

[1] B.A. Kolachev, I.S. Polkin, V.D. Talalaev, Titanium Alloys of Various Countries, Handbook, Moscow, VILS, 2000 (in Russian).

[2] V.M. Ilyenko, R.E. Shalin, Titanium alloys for aircraft gas turbine engines, Titanium 1 (1995) 23-29 (in Russian).

[3] I.P. Semenova, G.S. Dyakonov, G.I. Raab, Y.F. Grishina, Y. Huang, T.G. Langdon, Features of duplex microstructural evolution and mechanical behavior in the titanium alloy processed by equal-channel angular pressing, Adv. Eng. Mater. 20 (2018) 1700813, https://doi.org/10.1002/adem.201700813.

[4] I.M. Modina, A.V. Polyakov, G.S. Dyakonov, T.V. Yakovleva, A.G. Raab, I.P. Semenova, Peculiarities of microstructure and mechanical behavior of VT8M-1 alloy processed by rotary swaging, IOP Conf. Series 461 (2018), https://doi.org/ 10.1088/1757-899X/461/1/012056 012056.

[5] T.V. Yakovleva, G.S. Dyakonov, A.G. Stotskiy, I.P. Semenova, Microstructure and mechanical properties of workpieces of the ultrafine-grained two-phase Ti alloy produced by die forging, IOP Conf. Series 672 (2019), https://doi.org/10.1088/ 1757-899X/672/1/012065 012065.

[6] G. Dyakonov, T.V. Yakovleva, A. Stotskiy, A. Ibatullin, I. Semenova, Evolution of the microstructure and mechanical properties of the ultrafine-grained VT8M-1 during isothermal die forging and thermal treatment, Mater. Sci. Forum 1016 (2021) 418-422, https://doi.org/10.4028/www.scientific.net/MSF.1016.418.

[7] I. Weiss, F.H. Froes, D. Eylon, G.E. Welsch, Modification of alpha morphology in Ti-6Al-4V by thermomechanical processing, Metall. Mater. Trans. A 17 (11) (1986) 1935-1947, https://doi.org/10.1007/BF02644991.

[8] S. Li, Orientation stability in equal channel angular extrusion. Part II: Hexagonal close-packed materials, Acta Mater. 56 (5) (2008) 1031-1043, https://doi.org/ 10.1016/j.actamat.2007.11.003. 